\title{
Continuity of partially ordered soft sets via soft Scott topology and soft sobrification
}

\author{
A. F. Sayed \\ Mathematics Department, Al-Lith University College, Umm Al-Qura University P.O. Box 112, \\ Al-Lith 21961, Makkah Al Mukarramah, Kingdom of Saudi Arabia \\ E-mail address: dr.afsayed@hotmail.com
}

\begin{abstract}
Keywords: Continuous posset; Continuous soft domain; Embedded soft basis; round soft ideal completion; soft Sobrification; soft Scott topology.
\end{abstract}

\begin{abstract}
This paper, based on the concept of partially ordered soft sets (possets, for short) which proposed by Tanay and Yaylali [23], we will give some other concepts which are developing the possets and helped us in obtaining a generalization of some important results in domain theory which has an important and central role in theoretical computer science. Moreover, We will establish some characterization theorems for continuity of possets by the technique of embedded soft bases and soft sobrification via soft Scott topology, stressing soft order properties of the soft Scott topology of possets and rich interplay between topological and soft order-theoretical aspects of possets. We will see that continuous possets are all embedded soft bases for continuous directed completely partially ordered soft set (i.e., soft domains), and vice versa. Thus, one can then deduce properties of continuous possets directly from the properties of continuous soft domains by treating them as embedded bases for continuous soft domains. We will see also that a posset is continuous if its soft Scott topology is a complete completely distributive soft lattice.
\end{abstract}

\section{Introduction}

Soft set theory was first proposed by Molodtsov [19] in 1999. It is a general mathematical tool fordealing with uncertainties and not clearly defined objects. Traditional tools for formal modeling, reasoning and computing are crisp, deterministic and precise in nature. However, in most cases, complicated problems in economics, engineering, environment, social science, medical science, etc., that involved data which are not always all crisp cannot be successfully dealt with using classical methods because of various types of uncertainties present in these problems. Soft set is one of the various non-classical methods that can be considered as a mathematical tool for dealing with uncertainties.

Domain theory was initiated by Scott [21] in the early 1970s, and developed further by many researchers as a branch of mathematics that studies special kinds of parti ally ordered sets (posets) commonly called domains. Consequently, domain theory can be considered as a branch of order theory. The field has major applications in computer science, where it is used to specify denotational semantics, especially for functional programming languages.

Moreover, domain theory has evolved until became not only an important tool for applications in Computer Science, but also an exciting field of ongoing research in pure mathematics. There has been a rather rich and varied interplay between the theory of domains and topology. Several works, see for example (ch.14 of [11], [12, 13, 14, 15, 16, 17], [18], [7, 8, 9], [6] and [1]) documented evolution of domain theory and its relationships to topology that have had the greatest utility and important from the mathematical and theoretical computer Science sides.

This paper, based on the concept of partially ordered soft sets (possets, for short) which proposed by Tanay and Yaylali [23], we will give some other concepts which are developing the possets and helped us in obtaining a generalization of some important results in domain theory which has an important and central role in theoretical computer science. Moreover, We will establish some characterization theorems for continuity of possets by the technique of embedded soft bases and soft sobrification via soft Scott topology, stressing soft order properties of the soft Scott topology of possets and rich interplay between topological and soft order-theoretical 
aspects of possets. We will see that continuous possets are all embedded soft bases for continuous directed completely partially ordered soft set (i.e., soft domains), and vice versa. Thus, one can then deduce properties of continuous possets directly from the properties of continuous soft domains by treating them as embedded bases for continuous soft domains. We will see also that a posset is continuous if its soft Scott topology is a complete completely distributive soft lattice

\section{Preliminaries and basic definitions}

In this section, we first recall the basic definitions related to soft sets and ordered soft sets which would be used in the sequal.

Definition 2.1. [19] Let $U$ be an initial universe, $E$ be the set of parameters. Let $P(U)$ be the set of all subsets of $U$ and $A$ be a subset of $E$. A pair $(F, A)$ is called a soft set over $U$ where $F: A \rightarrow P(U)$ is a set-valued function.

In other words, a soft set over $U$ is a parameterized family of subsets of the universe $U$. For $\varepsilon$ $\in A, A(\varepsilon)$ may be considered as the set of $\varepsilon$-approximate elements of the soft set $(F, A)$. Clearly, a soft set is not a classical set. For illustration, Molodtsov considered several examples in [12].

Definition 2.2. [10] The soft set $(F, A)$ for every $\varepsilon \in A, F(\varepsilon)=\phi$.

Definition 2.3. [20] The soft set $(F, A)$ over $U$ is said to be a Null soft set denoted by $\Phi$, if over $U$ is said to be an absolute soft set if $F(e)=U$, for all $e \in A$ and is denoted by $\Psi$.

Definition 2.4. [4] The soft complement $(F, A)^{c}$ where $F^{c}(x)=U-F(x), \forall x \in A$.. of $(F, A)$ is defined by $(F, A)=\left(F^{C}, A\right)$

Definition 2.5. [10] For two soft sets $(F, A)$ and $(G, B)$ over a common universe $U$, we say that $(F, A) F$ is a soft subset of $(G, B)$ and is denoted by $(F, A) \subset(G, B)$ if

(i) $F \subseteq B$ and,

(ii) $\forall \varepsilon \in A, F(\varepsilon)$ and $G(\varepsilon)$ are identical approximations, which means $F(\varepsilon)=G(\varepsilon)$.

Definition 2.6. [10] Let $(F, A)$ and $(G, B)$ be two soft sets over a common universe $U$. The intersection of $(F, A)$ and $(G, B)$ is defined to be the soft set $(H, C)$ satisfying the following conditions:

(i) $\quad C=A \cap B$ and,

(ii) $\forall e \in C, H(e)=F(e) \cap G(e)$.

In this case, we write $(F, A) \cap(G)=,(H, C)$.

Definition 2.7. [10] Let $(F, A)$ and $(G, B)$ be two soft sets over a common universe $U$. The union of $(F, A)$ and $(G, B)$ is defined to be the soft set $(H, C)$ satisfying the following conditions:

(i) $\quad C=A \cup B$ and,

(ii) $\forall e \in C$,

$$
H(e)= \begin{cases}F(e) & \text { if } e \in A \backslash B ; \\ G(e) & \text { if } e \in B \backslash A ; \\ F(e) \cup G(e) & \text { if } e \in A \cap B .\end{cases}
$$

In this case, we write $(F, A) \tilde{\cup}(G, B)=(H, C)$. 
Definition 2.8. [25] Let $\tau$ the collection of soft sets o ver $X$. Then $\tau$ is called a soft topology on $X$ if it satisfies the following axioms:

(i) $\Phi, \Psi$ belong to $\tau$,

(ii) the union of any number of soft sets in $\tau$ belong to $\tau$,

(iii) the intersection of any two of soft sets in $\tau$ belong to $\tau$.

The triple $(X, \tau, A)$ is called a soft topological space over $X$. the members of $\tau$ is said to be soft open in $X$, and the soft set $(F, A)$ is called closed in $X$ if its relative component $(F, A)^{c}$ belong to $\tau$. The family of soft closed sets is denoted by $\tau^{c}$.

Definition 2.9. [22] Let $(X, \tau, A)$ be a soft topological space. The soft closure $C l(F, A)$ of a soft set $(F, A)$ is the soft set

$$
\tilde{\cap}\left\{(Q, A) \in \tau^{c}:(F, A) \subset(Q, A) .\right.
$$

Definition 2.10. [24] Let $(F, \tau, X),(G, v, Y)$ be soft topological spaces and let $T:(F, \tau, X) \rightarrow(G, v, Y)$ be a soft mapping. $T$ is said to be a soft continuous mapping (with respect to the soft topologies $\tau$ and $v$ ) if for each $V \in v, T^{-1}(V) \in \tau$ (i.e., the inverse of a soft open set a soft open set).

Definition 2.11. [2] Let $(F, A)$ and $(G, B)$ be two soft sets over $U$, then the cartesian product of $(F, A)$ and $(G, B)$ is defined as, $(F, A) \times(G, B)=(H, A \times B)$ where $H: A \times B \rightarrow P(U \times U)$

and $H(a, b)=F(a) \times G(b)$, where $(a, b)=A \times B$ i.e.

$H(a, b)=\left\{\left(h_{i}, h_{j}\right) \mid h_{i} \in F(a), h_{j} \in G(b)\right\}$

Definition 2.12. [2] Let $(F, A)$ and $(G, B)$ be two soft sets over $U$, then a relation $R$ from $(F, A)$ to $(G, B)$ is a soft subset of $(F, A) \times(G, B)=(H, A \times B)$.

In other words, a relation $R$ from $(F, A)$ to $(G, B)$ is of the form $R=\left(H_{1}, S\right)$ where $S \subset A \times B$ and $H_{1}(a, b)=H(a, b)$ for all $(a, b) \in S$ where $(H, A \times B)=(F, A) \times(G, B)$.

Definition 2.13. [2] Let $R$ be a relation on $(F, A)$, then

(1) $R$ is reexive if $H_{1}(a, a) \in R, \forall a \in A$.

(2) $R$ is symmetric if $H_{1}(a, b) \in R, \Rightarrow H_{1}(b, a) \in R$.

(3) $R$ is transitive if $H_{1}(a, b) \in R, H_{1}(b, c) \in R \Rightarrow H_{1}(a, c) \in R$ for every $a, b, c \in A$.

Definition 2.14. [3] A binary soft set relation $R$ on $(F, A)$ is an antisymmetric if $F(a) \times F(b) \in R$ and $F(a) \times F(b) \in R$ for every $F(a), F(b) \in(F, A) \Rightarrow F(a)=F(b)$.

Definition 2.15. ( [3], [5]) A binary soft set relation $R$ on $(F, A)$ which is reexive, antisymmetric and transitive is called a partial ordering of $(F, A)$.

The triple $(F, A, \leq)$ is called a partially ordered soft set or posset i.e a posset consists of two things, a soft set $(\mathrm{F}, \mathrm{A})$ and the partial ordering $\leq$

Suppose $(F, A, \leq)$ is a posset. Then, we write the statement:

$\mathrm{F}(\mathrm{a}) \leq \mathrm{F}(\mathrm{b})$ and is read " $\mathrm{F}(\mathrm{a})$ precedes $\mathrm{F}(\mathrm{b})$ " and also

$F(b) \geq F(a)$ means $F(a) \leq F(b)$ reads " $F(b)$ succeeds $F(a)$ ".

Definition 2.16. ( [3], [5] Let $\leq$ be an ordering of $(F, A)$ and $F(a)$ and $F(b)$ be any two elements in $(F, A)$. We say that $F(a)$ and $F(b)$ are comparable in the ordering if $F(a) \leq F(b)$ or $F(b) \leq F(a)$. We say that $F(a)$ and $F(b)$ are incomparable if they are not comparable. 
Definition 2.17. [23] Let $B \subset A$ and $(G, B) \subset(F, A)$ where $(\mathrm{F}, \mathrm{A})$ is ordered by $\leq .(G, B)$ is a chain in $(\mathrm{F}, \mathrm{A})$ if any two elements in $(G, B)$ are comparable.

Definition 2.18. [3] Let $(G, B, \leq)$ be a partially ordered soft set. Then,

a) For $b \in B, G(b)$ is the least element of $(G, B)$ in the ordering $\leq$ if $\forall x \in B, G(b) \leq G(x)$.

b) For $b \in B, G(b)$ is a minimal element of $(G, B)$ in the ordering $\leq$ if there exists no $x \in B$ such that $G(x) \leq G(b)$ and $G(x) \neq G(b)$.

a') For $b \in B, G(b)$ is the greatest element of $(G, B)$ in the ordering $\leq$ if $\forall x \in B, G(x) \leq G(b)$

b) For $b \in B, G(b)$ is a maximal element of $(G, B)$ in the ordering $\leq$ if there exists no $x \in B$ such that $G(b) \leq G(x)$ and $G(x) \neq G(b)$.

Definition 2.19. [23] Let $\leq$ be an ordering of $(F, A)$, let $(G, B) \subset(F, A)$.

- For $a \in A, F(a)$ is a lower bound of $(G, B)$ in the ordered soft set $(F, A, \leq)$ if $\leq \mathrm{G}(\mathrm{x}), \forall \mathrm{x} \in \mathrm{B} . F(a)$

- For $a \in A, F(a)$ is called in_mum of $(G, B)$ in $(F, A, \leq)$ (or the greatest lower bound) if it is the greatest element of the set of all lower bounds of $(G, B)$ in $(F, A, \leq)$.

Similarly,

- For $a \in A, F(a)$ is an upper bound of $(\mathrm{G}, \mathrm{B})$ in the ordered soft set $(F, A, \leq)$ if $G(x) \leq F(a), \forall x \in B$.

- For $a \in A$, it $F(a)$ is called supremum of $(G, B)$ in $(F, A, \leq)$ (or the least upper bound) if is the least element of the set of all upper bounds of $(G, B)$ in $(F, A, \leq)$.

We will write by the symbols $\vee$ and $\wedge$ instead of supremum and infimum, respectively.

Definition 2.20. [23] Consider a soft set $(F, A)$ equipped with reexsive, transitive relation $\leq$. This relation is called preorder and $(F, A)$ is a preordered soft set.

Definition 2.21. [23] Let $(F, A)$ be a soft set. $(F, A)$ is called a finite soft set, if it is a soft set with a finite parameter set.

Definition 2.22. [23] Let $(F, A)$ be a preordered soft set. A soft subset $(G, B)$ of $(F, A)$ is directed provided it is nonnull soft set and every finite soft subset of $(G, B)$ has an upper bound in $(G, B)$.

Definition 2.23. [23] Let $(F, A)$ be a soft set with a preorder $\leq$. For $(\mathrm{G}, \mathrm{B}) \subset(\mathrm{F}, \mathrm{A})$

i) $\downarrow(G, B)=(H, C)$ where $C=\{\mathrm{a} \in A: F(a) \leq G(b)$ for some $b \in B\}$ and $\mathrm{H}=\left.\mathrm{F}\right|_{\mathrm{C}}$.

ii) $(G, B)=(K, D))$ where $C=\{\mathrm{a} \in A: G(b) \leq F(a)$ for some $b \in B\}$ and $\mathrm{k}=\left.\mathrm{F}\right|_{\mathrm{D}}$.

iii) $(G, B)$ is a lower soft set iff $((G, B)=\downarrow(G, B)$.

iv) $(\mathrm{G}, \mathrm{B})$ is an upper soft set iff $(G, B)=(G, B)$.

v) $(G, B)$ is an ideal iff it is a directed lower soft set.

Remark 2.24. In the last definition; if $B=\{b\}$ then we will write $\downarrow(G, B)=\downarrow G(b)$ and $(G, B)=G(b)$, for simplicity.

Definition 2.25. [23] A partially ordered soft set is said to be a directed complete soft set if every directed soft subset has a supremum. 
Definition 2.26. [23] Let $(F, A)$ be a directed complete partially ordered soft set and $(G, B) \subset(F, A)$.

Then $(G, B)$ is called a Scott open soft set iff the following two conditions are satisfied:

i) $(G, B)=(G, A)$

ii) $\vee(D, C) \in(G, B)$ implies $(D, C) \cap(G, B) \neq \Phi$ for all directed complete soft sets $(G, B) \subset$ $(F, A)$.

Definition 2.27. [23] The collection $\tau$ of all Scott open soft sets of $(F, A)$ is called soft Scott topology on $(F, A)$.

Definition 2.28. [23] Let $(F, A, \tau)$ be a soft Scott topology, and $(G, B) \subset(F, A)$. Then $(G, B)$ is called Scott closed soft set if $(\mathrm{G}, \mathrm{B})^{\mathrm{c}}$ is Scott open soft set.

A partially ordered set or poset [6] is a nonempty set together with an equivalence relation; i.e. reflexsive, antisymmetric and transitive relation.

Definition 2.29. [20] Let A none-empty poset $L$ is called a lattice if for all $x, y \in L ; x \vee y$ and $x \wedge y$ exist, It is denoted by $(L, \vee, \wedge)$ or $L$ for simplicity

Definition 2.30. [20] A nonempty subset $M$ of $L$ is said to be a sublattice of $L$ if $a, b \in M$ implies $a \vee b, a \wedge b \in M$.

Definition 2.31. [20] Let $(F, A)$ be a soft set over L. Then $(F, A)$ is said to be a soft lattice over $L$ if $F(x)$ is a sublattice of $L$, for all $x \in A$.

\section{Continuous partially ordered soft sets}

Definition 3.1. Let $(F, A)$ and $(G, B)$ be possets. A function $f:(F, A) \rightarrow(G, B)$ is called soft monotone if for all $F(x), F(y) \in(F, A)$ with $F(x) \leq F(y)$ we also have $f[F(x)] \leq f[F(y)]$ in $(G, B)$.

A one-one function $f:(F, A) \rightarrow(G, B)$ where both $f$ and $f^{-1}$ are soft monotone is called order soft isomorphism.

Definition 3.2. Let $(F, A)$ and $(G, B)$ be possets. A function $f:(F, A) \rightarrow(G, B)$ is soft Scott-continuous if it is soft monotone and if for each directed soft subset $(H, C)$ of $(F, A)$ we have

$f(\vee(H, C))=\vee f(H, C)$.

Definition 3.3. Let $(F, A)$ be a posset. For any two elements $F(x), F(y) \in(F, A) . F(x)$ is approximate to $F(y)$, and write $F(x) F(y)$, if for any directed soft subset $(G, B) \subset(F, A)$ with $\vee(G, B)$ existing and $F(y) \leq \vee(G, B)$, there exists $G(z) \in(G, B))$ such that $F(x) \leq G(z)$.

An element $F(x) \in(F, A)$ satisfying $F(x) F(x)$ is called compact. The set of compact elements of $(F, A)$ is denoted as $K(F, A)$

We introduce the following notation for $F(x), F(y) \in(F, A)$ and $(G, B) \subset(F, A)$ :

$$
\begin{aligned}
& \Downarrow F(x)=\{F(y) \in(F, A) \mid F(y) \ll F(x)\} \\
& \Uparrow F(x)=\{F(y) \in(F, A) \mid F(x) \ll F(y)\} \\
& \Uparrow(F, A)=\bigcup_{F(a) \in(F, A)} \Uparrow F(a) .
\end{aligned}
$$

Proposition 3.4. If $(F, A)$ is a continuous posset, then the approximation relation $<<$ has the interpolation property:

$F(x)<<F(z) \Rightarrow \exists F(y) \in(F, A)$ such that $F(x)<<F(y)<<F(z)$. 
Proof. Define $(G, B)=\left\{G(u): \exists F(y) \in(F, A)\right.$ such that $G(u)<<F(y)<<F(z) . G=\left.F\right|_{B}$. It can be easily deduced from the directedness soft and the approximation relation property of $\Downarrow F(p)$ for every $F(p) \in(F, A)$ that $(G, B)$ is directed soft and has $F(z)$ as its least upper bound. Thus it follows from $F(x)<<F(z)$ that there is some $G(u) \in(G, B)$ such that $\mathrm{F}(\mathrm{x}) \leq \mathrm{G}(\mathrm{u})$. By the construction of $(G, B)$, there is some $F(y) \in(G, B)$ such that $F(x) \leq G(u)<<F(y)<<F(z)$, as desired.

Definition 3.5. A posset $(F, A, \leq)$ is called a continuous posset (resp., an algebraic posset) if for each $F(x) \in(F, A)$, the set $\{F(y) \in(F, A): F(y) F(x)\}$ (resp., $\downarrow F(x) \cap K(F, A)$ ) is a directed soft set and $F(x)=\vee\{F(y) \in(F, A): F(y) F(x)\}$ (resp., $F(x)=\vee\{\downarrow F(y) \cap K(F, A)\})$.

Definition 3.6. A directed complete posset which is continuous as a posset is called a soft domain

Definition 3.7. A soft lattice $(F, A)$ over $L$ is complete if is a partially ordered soft set in which all soft subsets have both a supremum and an infimum .

Definition 3.8. A continuous posset which is also a complete soft lattice is called a continuous soft lattice.

If $(G, B)$ is a soft subset of a posset $(F, A)$ and $(H, C)$ is a directed soft subset of $(G, B)$,then for every element $F(a) \in(F, A)$. We will write $(H, C)_{F(a)}=\Downarrow F(a) \cap(H, C)$. In this case, we call elements of $(H, C)_{F(a)}$ approximate to $F(a)$ relative to $(H, C)$.

Definition 3.9. Let $(F, A)$ be a posset and $(G, B) \subset(F, A),(G, B)$ is called a soft basis for $(F, A)$ if $\forall F(a) \in(F, A)$, there is a directed soft set $(H, C)_{F(a)} \subset(G, B)$ such that $\forall H(d) \in(H, C)_{F(a)}, H(d)<<_{(F, A)} F(a)$ and $\vee_{(F, A)}(H, C)_{F(a)}=F(a)$, where the subscript $(F, A)$ means to take relevant operations in posset $(F, A)$.

\subsection{Embedded soft bases}

Definition 3.10. Let $(F, A),(G, B)$ be a possets. If there is a map $j:(G, B) \rightarrow(F, A)$ satisfying

(1) $j$ preserves existing directed soft supremums;

(2) $j:(G, B) \rightarrow(H, j(B))$ is an order soft isomorphism, where $H=F_{j(B)}$;

(3) $j(G, B)$ is a soft basis .

Then $((G, B), j)$ is called an embedded soft basis for $(F, A)$. If $(G, B) \subset(F, A)$ and $((G, B), j)$ is an embedded soft basis for $(F, A))$, where $\mathrm{i}$ is the inclusion map, then we say also that $(G, B)$ is an embedded soft basis for $(F, A)$.

It is easy to see that if $(G, B) \subset(F, A)$, then $(G, B)$ is an embedded soft basis for $(F, A)$ iff $(G, B)$ is a soft basis for $(F, A)$ and for every directed soft set $(H, C) \subset(G, B)$ with existing $\vee_{(G, B)}(H, C)$, one has $\vee_{(G, B)}(H, C)=\vee_{(F, A)}(H, C)$. We can also observe that if $((G, B), j)$ is an embedded soft basis for $(F, A)$, then $j(G, B) \subset(F, A)$ is an embedded soft basis for $(F, A)$.

Proposition 3.11. If $(G, B)$ is an embedded soft basis for $(E, T)$, then for all

$G(x), G(y) \in(G, B), G(x)<<_{(G, B)} G(y)$ if and only if $G(x)<<_{(E, T)} G(y)$.

Proof. $\Rightarrow$ : Let $G(x), G(y) \in(G, B)$ with $G(x)<<_{(G, B)} G(y)$. Let $(H, C)_{G(y)} \subset(G, B)$ be the existing directed soft set in Definition 3.9. For a directed soft subset $(H, C) \subset(G, B)$ with $\vee_{(E, T)}(H, C) \geq F(y)$, since $\vee_{(G, B)}(H, C)_{G(y)}=\vee_{(E, T)}(H, C)_{G(y)}=G(y) \in(G, B)$, there is some $H(b) \in(H, C)_{G(y)} \subset(G, B)$ such that $G(x) \leq G(b)$. Thus by $H(b) \in(H, C)$ and $H(b) G(x)<<_{(E, T)} G(y)$, there is some such that $G(x) \leq H(b) \leq H(d)$, showing that $G(\mathrm{x})<{ }_{(E}$ $, T)^{\cdot} G(y)$ 


\section{$\Leftarrow$ : Let $F(x), F(y) \in(F, A)$ with $F(x) F(y)$. Let $(H, C) \subset(F, A)$ with} $\vee_{(F, A)}(H, C) \geq F(y)$. Then by Definition $3.10(1) ; \vee_{(E, T)}(H, C)=\vee_{(F, A)}(H, C) \geq F(y)$. Thus, by $F(x)<<_{(E, T)} F(y)$, there is $\in(H, C)$ such that $F(x) \geq H(d)$. This shows that $F(x)_{(F, A)} F(y)$, as desired.

Proposition 3.12. If $(G, B)$ is a soft basis for a posset $(F, A)$, then $(F, A)$ is continuous; if $(G, B)$ is an embedded soft basis for $(F, A)$ (up to a soft isomorphism), then $(G, B)$ itself is also continuous.

Proof. It is easy to see that if $(F, A)$ has a soft basis $(G, B)$, then $(F, A)$ is continuous. We next show that if $(G, B)$ is an embedded soft basis for $(F, A)$, then $(G, B)$ is also continuous.

Let $G(a) \in(G, B) \subset(F, A)$. Then $\Downarrow_{(F, A)} G(a)$ is a directed soft set and $\vee_{(F, A)} \Downarrow_{(F, A)} G(a)$ Let $(H, C)_{G(a)} \subset(G, B)$ be the directed soft set in Definition 3.9. Then $(H, C)_{G(a)}$ is a directed soft set in $(G, B)$ with existing $\vee_{(G, B)}(H, C)_{G(a)}=\vee_{(F, A)}(H, C)_{G(a)}=G(a) \in(G, B)$. By Proposition 3.11, we have for every $H(d)_{G(a)} \in(H, C)_{G(a)}, H(d)_{G(a)}<<_{(G, B)} G(a)$. Thus $(G, B)$ is a soft basis for itself, and by the first part of this proposition $(G, B)$ is continuous.

\subsection{Abstract soft bases and round soft ideal completions}

Definition 3.13. Let $((F, A), \quad)$ be a soft set equipped with a binary soft relation $\precsim$ The binary soft relation $\preceq$ is called fully transitive if it is transitive $(F(a) \preceq F(b), F(b) \preceq F(c)$ for every $F(a), F(b), F(c) \in(F, A)$ imply $F(a) \preceq F(c))$ and satisfies the strong interpolation property: $\forall|(G, B)|<\infty,(G, B) F(c) \Rightarrow \exists F(b) F(c)$, such that $(G, B) F(b)$, where $(G, B) \preceq F(b)$ means $\forall G(d) \in(G, B), G(d) \preceq F(b)$. If $((G, B)$, ) is a soft set equipped with a binary soft relation which is fully transitive, then $((G, B)$, ) is called an abstract soft basis.

Definition 3.14. Let $((G, B), \quad)$ be an abstract soft basis. A nonull soft subset $(M, I)$ of $(G, B)$ is a round soft ideal if

(1) $\forall M(y) \in(M, I), M(x) \preceq M(y) \Rightarrow M(x) \in(M, I)$;

(2) $\forall M(x), M(y) \in(M, I), \exists M(z) \in(M, I)$ such that $M(x) \preceq M(z)$ and $M(y) M(z)$.

The set of all round soft ideals of $(G, B)$ soft ordered by soft set inclusion is called the round soft ideal completion of $(G, B)$, denoted by $R S I(G, B)$.

Proposition 3.15. Let $((F, A), \preceq)$ be an abstract soft basis. Define map $j:(F, A) \rightarrow R S I(F, A)$ such that $\forall F(y) \in(F, A), j(F(y))=\downarrow \downarrow F(y):=\{F(x): \preceq F(x) F(y)\}$. Then $j(F, A)$ is a soft basis for $R S I(G, B)$, and thus $R S I(G, B)$ is a continuous soft domain.

Proposition 3.16. If $(F, A)$ is a continuous posset, then $((F, A), j)$ is an embedded soft basis for $R S I(F, A)$, where $j$ is of Proposition 3.15 for the abstract soft basis $((F, A),<<$.

Proof. By the proposition 3.15, $j(F, A)$ is a soft basis for $R S I(F, A)$. That $j$ is continuous and $j:(F, A) \rightarrow j(F, A) \subset R S I(F, A)$ is a soft isomorphism can be deduced from the continuity of $(F, A)$. So, by Definition $3.10(2)$, the corollary holds.

Theorem 3.17. Let $(F, A)$ be a poset. Then $(F, A)$ is continuous $((F, A), j)$ is an embedded soft basis for the round soft ideal completion $\operatorname{RSI}(F, A)$.

Proof. If $((F, A), j)$ is an embedded soft soft basis for the round ideal completion $R S I(F, A)$, then $j(F, A)$ is an embedded soft basis for $R S I(F, A)$. By Proposition 3.12, $(F, A) \cong j(F, A)$ and $R S I(F, A)$ are all continuous. 
Conversely, if $(F, A)$ is continuous, then by Proposition 3.16, we have that $((F, A), j)$ is an embedded soft basis for $R S I(F, A)$.

We now arrive at our first characterization theorem for the continuity of posets.

Theorem 3.18. A poset $(F, A)$ is continuous iff $(F, A)$ is soft isomorphic to an embedded soft basis for a directed completely partially ordered soft set.

Proof. If $(F, A)$ is continuous, then by Theorem 3.17, $(F, A) \cong j(F, A)$ is an embedded soft basis for the round soft ideal completion $R S I(F, A)$ which is a directed completely partially ordered soft set.

Conversely, if $(F, A)$ is soft isomorphic to an embedded soft basis for a directed completely partially ordered soft set $(H, C)$, then by Proposition $3.12,(F, A)$ is continuous.

\section{Characterization theorems by soft Scott topology and soft sobrification}

In this section, we introduce the concept of soft sobrification and study Characterization theorems for continuous partially ordered soft sets by soft Scott topology and soft sobrification.

Definition 4.1. A soft subset $(F, A)$ of a soft topological space $(X, \tau, A)$ is said to be irreducible if for any two soft closed subset $\left(G_{1}, B_{1}\right)$ and $\left(G_{2}, B_{2}\right)$ such that $(F, A) \subset\left(G_{1}, B_{1}\right) \cup\left(G_{2}, B_{2}\right)$, either $(F, A) \subset\left(G_{1}, B_{1}\right)$ or $(F, A) \subset\left(G_{2}, B_{2}\right)$.

The soft closure of any singleton soft subset is alwayes a soft closed irreducible subspace.

Definition 4.2. The soft topological space $(X, \tau, A)$ is said to be soft sober if every soft closed irreducible set $(C, A)$ is the soft closure of a unique soft point, $(F, A)=\{F(x)\}^{c}$ for an unique $F(x) \in(F, A)$.

Definition 4.3. Given any soft topological space $(X, \tau, A)$, A pair $\left((X, \tau, A)^{\mathrm{ss}}, \mathrm{j}\right)$ is called a soft sobrfication of $(X, \tau, A)$ if $(X, \tau, A)^{\mathrm{ss}}$ is a soft sober space and $\mathrm{j}:(X, \tau, A) \rightarrow(X, \tau, A)^{\mathrm{ss}}$ is a soft continuous mapping such that $\mathrm{j}^{-1}: S O(X, \tau, A)^{\mathrm{ss}} \rightarrow S O(X, \tau, A)$ is a soft lattice isomorphism from the soft lattice $S O(X, \tau, A)^{\mathrm{ss}}$ of soft open sets of $(X, \tau, A)^{\mathrm{ss}}$ onto the soft lattice $S O(X, \tau, A)$ of soft open sets of $(X, \tau, A)$.

Proposition 4.4. Let $(G, B)$ be an embedded soft basis for a continuous posset $(F, A)$. Then the inclusion map $i:(G, B) \rightarrow(F, A), \forall G(a) \in(G, B), i(G(a))=G(a)$ induces a soft isomorphism $i^{-1}: \tau_{(F, A)} \cong \tau_{(G, B)}$. As a consequence, $\tau_{(G, B)}=I{ }^{1} \tau_{(\mathrm{F}, \mathrm{A})}=\left\{(F, U) \cap(G, B):(F, U) \in \tau_{(\mathrm{F}, \mathrm{A})}(G, B)\right\}$ is the subspace soft topology.

Proof. Define $j: \tau_{(G, B)} \rightarrow \tau_{(F, A)}$ such that $\forall(G, M) \in \tau_{(G, B)}, j^{*}(G, M)=i(G, M)$.

(1) Claim that $i(G, M)$ is soft Scott open and $j^{*}$ is well defined. In fact, for all directed soft set $(H, D) \subset(F, A)$ with $\vee_{(F, A)}(H, D) \in i(G, M)$, there is some $G(g) \in(G, M)=\cup_{G(h) \in(G, M)} \Uparrow_{(G, B)} G(h)$ such that $G(g) \leq \vee_{(F, A)}(H, D)$. For this $G(g)$, there is some $G(h) \in(G, M)$ such that $G(h)_{(G, B)} G(g)$. By Proposition 3.11, we have

$G(h)<<_{(F, A)} G(g)$. Thus there is some $H(d) \in(H, D)$ such that $i(G(h))=G(h) \leq H(d)$ and $H(d) \in i(G, M)$, showing the soft Scott openness of $i(G, M)$.

(2) $j^{*}$ and $j^{-1}$ are both soft order preserving: Clear.

(3) $j^{*} i^{-1}=i d \tau_{(F, A)}$ : For all $(F, N) \in \tau_{(F, A)}$, clearly $j^{*} i^{-1}(F, N) \subset(F, N)$.

On the other hand, let $F(t) \in(F, N)$, then there is some $H(b) \in(H, D)$ such that $i(H(b))=H(b) \in(F, N)$, where $(H, D)_{F(t)} \subset(G, B)$ is the directed soft set as in Definition 3.9. 
Thus, $F(t) \geq H(b) \in i^{-1}(F, N)$ and $F(t) \in j^{*} i^{-1}(F, N)$, showing that $(F, N) \in j^{*} i^{-1}(F, N)$. $\left(4 i^{-1} j^{*}=i d \tau_{(G, B)}::\right.$ For all $(G, M) \in \tau(G, B)$, clearly $i^{-1} j^{*}(G, M) \supset(G, M)$. Conversely, let $H(b) \in i^{-1} j^{*}(G, M)$ then $H(b) \in i(H(b)) \in j^{*}(G, M)=i(G, M)$. Thus there is some $G(g) \in(G, M)$ such that $H(b)=i(H(b)) \geq i(G(g))=G(g)$. Since $(G, M)$ is an upper soft set in $(G, B), H(b) \in(G, M)$. showing $i^{-1} j^{*}(G, B) \subset(G, B)$

To sum up, $i^{-1}$ and $j^{*}$ are mutually inverse lattice soft isomorphisms, and $\tau_{(G, B)}$ are the images of $\left.\tau_{(F, A)}\right)$ under $I^{-1}$, as desired.

Corollary 4.5. If $(F, A)$ is a continuous posset and $R S I(F, A)$ its round soft ideal completion, Then $\left.j^{-1}: \tau_{R S I(F, A)} \rightarrow \tau_{(F, A)}\right)$ is a lattice soft isomorphism, where $j:(F, A) \rightarrow R S I(F, A)$ defined by $\forall F(x) \in(F, A), j(F(x))=\Downarrow F(x) \in R S I(F, A))$. As a consequence, $(R S I(F, A), j)$ is a soft sobrification of $(F, A)$ in the soft $\operatorname{Scott}$ topology whenever $(F, A)$ is continuous.

Proof. By Theorem 3.17, $(F, A)$ is an embedded soft basis for $\operatorname{RSI}(F, A)$. Then it follows from Proposition 4.4 that $j^{-1}: \tau_{R S I(F, A)} \rightarrow \tau_{(F, A)}$ is a lattice soft isomorphism.

Theorem 4.6. If $(F, A)$ is a continuous posset, then its soft Scott topology $\tau_{(F, A)}$ is a completely distributive soft lattice.

Proof. By Corollary 4.5, we have $\tau_{(F, A)} \cong \tau_{R S I(F, A)}$. Since $R S I(F, A)$ is a continuous directed completely partially ordered soft set, then $\tau_{R S I(F, A)}$ is a completely distributive soft lattice, as desired. .

With the above results, we immediately have our second characterization theorem:

Theorem 4.7. (The Characterization theorem) A posset $(F, A)$ is continuous iff its soft Scott topology is completely distributive.

Theorem 4.8. Let $(F, A)$ be a posset. Then $(F, A)$ is continuous $R S I(F, A)$ is continuous and with the soft Scott topology, $R S I((F, A), j)$ is a soft sobrification of $\left((F, A), \tau_{(F, A)}\right)$.

Proof. $\Rightarrow$ : If $(\mathrm{F} ; \mathrm{A})$ is continuous, then by Corollary $4.5 j^{-1} \tau_{R S I(F, A)} \cong \tau_{(F, A)}$., i.e., $(R S I(F, A), j)$ is a soft sobrification of $\left((F, A), \tau_{(F, A)}\right)$

$\Leftarrow$ : If $R S I(F, A)$ is continuous and $(R S I(F, A), j)$ is a soft sobrification of $\left((F, A), \tau_{(F, A)}\right)$, then $\tau_{(F, A)} \cong \tau_{R S I(F, A)}$ is completely distributive. By Theorem 4.7, $(F, A)$ is continuous.

\section{References}

[1] S. Abramsky and A. Jung, Domain theory, In S. Abramsky, D. M. Gabbay, and T. S. E. Maibaum, editors, Handbook of Logic in Computer Science, volume 3, Clarendon Press, (1994), 1-168.

[2] K. V. Babitha and J. J. Sunil, Soft set relations and functions, Computers and Mathematics with Applications 60 (2010), 1840-1849.

[3] K. V. Babitha and J. J. Sunil, Transitive closures and ordering on soft sets, Computers and Math- ematics with Applications 62 (2011), 2235-2239.

[4] N. Çgman, S. Karatas and S. Enginoglu, Soft topology, Computers and Mathematics with Applications 62, (2011), 351-258 .

[5] M. K. Dauda, I. Aliyu and A. M. Ibrahim, Partial Ordering in Soft Set Context, Mathematical Theory and Modeling, Vol.3, No.8, (2013), 97-100. 
[6] G. Gierz, K. H. Hofmann, K.Keimel, J. D.Lawson, M. Mislove and D. S. Scott, A Compendium of Continuous Lattices, Springer-Verlag, Berlin-Heidelberg- New York, 1980.

[7] J. D. Lawson, Spaces of maximal points, Math. Comput. Sci. 7 ,1, (1997), 543-555.

[8] J. D. Lawson, The upper interval topology, Property M, and Compactness, Electronic Notes in Theoretical Computer Science 13, (1998).

[9] J. D. Lawson, Encounters between topology and domain theory, International Colloquium on domain theory and its applications. October 17-25, (1999), Shanghai, China.

[10] P. K. Maji, R. Biswas and A. R. Roy, Soft set theory, Computers and Mathematics with Applications 45, (2003), 555-562.

[11] Martin, K., Mislove, M. W. and Reed, G. M., Topology and Domain Theory, Recent progress in General Topology II, (2002).

[12] K. Martin, Domain theoretic models of topological spaces, Proceedings of Comprox III, ENTCS, 13, (1998).

[13] K. Martin, Nonclassical Techniques for Models of computation, Topology Proceeding Summer Issue, 24, (1999).

[14] K. Martin, The spaces of maximal elements in a compact domain, Electronic Notes in theoretical computer science 40, (2000).

[15] K. Martin, A foundation for computation, Ph.D. Thesis, Tulane University, Department of Mathematics, 2000.

[16] K. Martin, Unique Fixed Points in Domain Theory, Electronic Notes in theoretical computer science 45, (2001).

[17] K. Martin, The regular spaces with countably based models, Theoretical computer science, volume 305, 18, Issues 1-3, (2003), 299-310.

[18] M. W. Mislove, Topology, Domain theory and theoretical Computer Science, Topology and its applications, 89, (1998), 3-59.

[19] D. A. Molodtsov, Soft set theory-First results, Computers and Mathematics with Applications 37, (1999), 19-31.

[20] E. K. R. Nagarjan and G. Meenambigai, An application of soft sets to lattices , Kragujevac Journal of Mathematics Vo.135, No.1, (2011), 75-87.

[21] D. Scott, Outline of the mathematical theory of computation, in: Proc. of the 4th Princeton Conf. on Information Science (1970), 169-176.

[22] M. Shabir and M. Naz, On soft topological spaces, Comput. Math. Appl., 61, (2011), 1786- 1799.

[23] B. Tanay, G. Yaylali, New structures on partially ordered soft sets and soft Scott topology, Annals of Fuzzy Mathematics and Informatics, 7, (1), (2014), 89-97.

[24] D. Wardowski, On a soft mapping and its fixed points, Wardowski Fixed Point Theory and Applications 2013, 2013:182. http://www.fixedpointtheoryandapplications.com/content/2013/1/182

[25] I. Zorlutuna, M. Akdag, W. K. Min, and S. Atmaca, Remarks on soft topological spaces, Annals of Fuzzy Mathematics and Informatics, 3, (2012), 171185. 